\title{
PROTOTIPO DE ESTACIÓN DE CARGA PARA BATERÍAS DE APARATOS ELECTRÓNICOS CON ENERGÍA RENOVABLE USANDO PANELES SOLARES PARA LA UNIVERSIDAD DE ORIENTE.
}

\section{CHARGING STATION PROTOTYPE FOR ELECTRONIC DEVICES USING RENEWABLE ENERGY FROM SOLAR PANELS FOR THE UNIVERSITY OF ORIENTE.}

\author{
Manfredy Alberto Molina Villegas | Ingeniero en Sistemas \\ Hernán José Hernández Durán | Arquitecto \\ Investigadores | Universidad de Oriente \\ mmolina@univo.edu.sv |hhernandez@univo.edu.sv
}

\section{Resumen}

Este proyecto pretende establecer un prototipo de estación de carga a partir del uso de panel solar como propuesta de energía limpia ante los múltiples problemas ambientales por el cambio climático; aprovechando la abundante luz solar de la ciudad de San Miguel, El Salvador. La estación de carga solar se diseñó como un quiosco para mayor comodidad de sus usuarios, con una mesa de madera de $1.5 \mathrm{~m} 2,6$ sillas de madera, un techo de $2.4 \times 0.30$ m2 de lámina zinc alum, una caja cuadrada - en el centro de la mesa- donde encajan 4 tomacorrientes dobles y 4 puertos USB. En la parte eléctrica y fotovoltaica, se hicieron diferentes cálculos para determinar la cantidad y potencia de los materiales y equipos. Se realizó el cálculo estimado de consumo de los dispositivos que se conectarían al cargador solar, 4 tomacorrientes dobles, 2 luces led y un sensor de movimiento, luego se calcularon aspectos técnicos como cantidad y potencia del panel solar que se necesitaría y el rendimiento general de equipos a utilizar. El sistema fotovoltaico ilumina de manera autónoma al no recibir luz solar y detectar el movimiento de personas. El prototipo aporta, un ahorro de energía en la red eléctrica de la Universidad de Oriente y brinda beneficios de conectividad a estudiantes, docentes, visitantes en el campus.

Los resultados han sido favorables con la estación de carga; con el aprovechamiento de la luz natural del sol, sin afectar el medio ambiente, se fomenta el uso de las energías renovables $y$, a su vez, ha tenido una demanda bastante alta por parte de sus usuarios. Esto es indispensable para combatir el cambio climático, pues aporta, a su vez, un ahorro de energía de la red eléctrica para la Universidad de Oriente. 
Palabras clave: Energías renovables, medio ambiente, sistema fotovoltaico, cambio climático, panel solar, energía limpia.

\section{Abstract}

This project presents a prtotype of a charging station using solar panels as a proposal for clean energy, attempting to reduce the negative effects of climate change; taking advantage of the abundant sunlight in the city of San Miguel, El Salvador. The charging station was design as a kiosk for the commodity of the users, with a study table of $1.5 \mathrm{~m} 2,6$ different wood seats, a zinc alum roof of $2.4 \times 0.30 \mathrm{~m} 2$, and a plug socket -in the middle of the tablewith 4 plugs and 4 USB ports. In the electric and photovoltaic components, different calculations were made to determine the quantity and power of the materials and equipment. An estimated calculation of the consumption of the possible devices connected was made (4 power outlets, 2 led lights and a movement sensor). Other technical aspects were calculated afterwards, such as the quantity and power that the solar panel would need and the general performance of the equipment. The photovoltaic system lights up autonomously when movement is detected. This prototype contributes to energy saving in the electric system of Universidad de Oriente and facilitates connectivity among students, teachers and campus visitors.

Keywords: Renewable energies, environment, photovoltaic system, climate change, solar panel, clean energy.

\section{Introducción}

Cuando la energía se consigue a través de fuentes naturales inagotables, se denomina “energía renovable". Son inagotables o bien porque contienen una gran cantidad de energía o bien porque son capaces de regenerarse por medios naturales.

Utilizar más las energías renovables nos ayudaría a solucionar muchos problemas ambientales como el cambio climático, que ha permitido que este tipo de energías se posicionen en el mundo como una importante alternativa, ya que son fuentes de energía limpia, inagotables y de creciente competitividad, debido a factores como el aumento de la rentabilidad de las tecnologías renovables, iniciativas de política aplicada, un mejor acceso al financiamiento, la seguridad energética y cuestiones de medio ambiente.

De esta manera nació la necesidad crear el proyecto de un prototipo de estación de carga para baterías de aparatos eléctricos y electrónicos usando paneles solares. La estación de carga solar es una fuente autónoma de energía renovable; esta utiliza energía solar 
fotovoltaica (panel solar) — que proviene del sol—, captura dicha energía y recarga la batería interna del dispositivo, para más tarde poder alimentar cualquier otro dispositivo como teléfonos, tablets, iPads, laptops, entre otros.

El cargador solar estará diseñado en forma de quiosco, con mesa de madera de $1.5 \mathrm{~m} 2,6$ sillas de madera, techo de $2.4 \times 0.30 \mathrm{~m} 2$ de lámina zinc alum, 4 tomacorrientes dobles, 4 puertos USB, 2 luces led, un panel solar de 350 watts, entre otros materiales eléctricos que permitan su buen funcionamiento. Además, el sistema fotovoltaico posee la autonomía de encender sus luminarias cuando este no reciba luz solar, a través de unas fotoceldas, y detecta movimientos de personas; de esta forma, se ahorran las cargas eléctricas de la batería.

Así, se fomenta el uso de las energías renovables, que ha tenido una demanda bastante alta por parte de sus usuarios, por su llamativo diseño de mesa y sillas de madera en su estructura. Además de ser capaz de satisfacer al $100 \%$ como fuente de energía renovable, por ser limpia e inagotable, indispensable para combatir el cambio climático. Ello aporta, a su vez, un ahorro de energía a la red eléctrica de la Universidad de Oriente.

\section{Materiales y Métodos}

El proyecto se inició en mayo de 2019 y finalizó en marzo de 2020. La implementación se desarrolló en la Universidad de Oriente, sede central, en la 4. ${ }^{\mathrm{a}}$ calle poniente, San Miguel, EI Salvador.

\section{Recopilación de la información de energía fotovoltaica}

Para dar inicio al proyecto, se recopiló información bibliográfica, sitios web, artículos y documentos especializados relacionados con la energía renovable, eléctrica, normativas de electricidad y temas a fines. Se buscaron proyectos de energías renovables para comprender su funcionamiento modular. También se incluyó a seis alumnos de la carrera de Eficiencia Energética como apoyo para aplicar sus conocimientos aprendidos y para que ellos ejecutaran el ensamblaje del sistema fotovoltaico. $Y$ para aclarar todas las dudas sobre sistemas eléctricos y fotovoltaicos, se tuvo a un docente consultor, experto en energía renovable y con amplia experiencia en la parte eléctrica.

\section{Diseño del cargador solar}

Luego de recopilar toda la información, y de haber recibido asesoría del consultor experto, se realizó un diseño de estación de carga solar, resistente, para que de él pudieran hacer uso los alumnos, visitantes, personal docente y administrativo. 
Figura I. Diseño de la estación de carga solar en forma de quiosco, con un tubo chapa 14 como base, mesa de madera, 6 sillas de madera y una caja cuadrada para los tomacorrientes. Funcionamiento nocturno por las luces led.



Fuente: Elaboración propia

\section{Cotización de materiales de construcción, eléctricos y fotovoltaicos}

Luego de realizar el diseño, se hizo un listado de materiales para la construcción del quiosco, para luego hacer las cotizaciones con los proveedores y ver cuál era la mejor opción de compra. Así mismo, se buscó a la empresa que se encargaría de realizar la mano de obra para la construcción del quiosco.

De igual forma, se hizo un listado de los materiales eléctricos para hacer las cotizaciones a los proveedores; la implementación del sistema eléctrico fotovoltaico la ejercerían los alumnos de Eficiencia Energética, luego de recibir capacitaciones por parte del consultor certificado en energía fotovoltaica.

Figura 2. Plano arquitectónico o plano de construcción de la estación de carga solar con toda la información necesaria que se ha de seguir para poder construir el proyecto.






\section{Construcción del quiosco}

La construcción se realizó frente al auditorio de la Universidad de Oriente. Está entre los pasillos de las salas de docentes de las Facultades de Humanidades, Ciencias Jurídicas y Economía, así como la sala de defensa de tesis y la Dirección de Investigación. Las medidas necesarias se calcularon desde el centro de la baldosa, donde se colocó el tubo base de chapa 14" para la base del quiosco.

\section{Zapata para la cimentación}

Se utilizó una zapata, que es un tipo de cimentación artificial que sirve para darle mayor resistencia a compresiones. Esta quedó situada en el pilar de la estructura. Se rellenó con concreto la ubicación donde está la zapata para darle mayor resistencia al tubo galvanizado de 4 " chapa I4, la cual será la base del quiosco. La profundidad para encajar la zapata es de I m2 y su tamaño es de $0.8 \mathrm{~m}$ en cada lado.

Se rellenó con concreto la ubicación donde está la zapata para darle mayor resistencia al tubo galvanizado de 4" chapa I4, la cual será la base del quiosco. Una de las soluciones clásicas para la cimentación es la realización del pedestal para darle mayor estabilidad y protección al tubo base, por la necesidad de soportar bastante peso en la estación de carga como el techo, el panel solar, la caja marina (con la batería, el control de carga y el inversor).

Luego de haber hecho con concreto el pilote, se reconstruyó la baldosa, dejando un espacio para colocar el cepo con la barra copper, que le dará el polo a tierra al sistema eléctrico para una mayor protección.

\section{Estructura del techo}

La construcción de la estructura del techo metálico se realizó con tubo galvanizado de $2 x \mathrm{I}$ " chapa 14 para las diagonales y para la base horizontal se empleó tubo galvanizado de $|x|$ " chapa 14 de $1 \mathrm{~m}$ a partir del tubo base. La ventaja del techo metálico es que tiene las propiedades de durabilidad, economía, resistencia, bajo normas de calidad, lo que lo hace uniforme en cuanto a dimensiones y resistencia. La construcción del techo se realizó con lámina acanalada de aluminio y capote zinc alum, elegida por su durabilidad, facilidad de instalación, económicamente barata y mantenimiento mínimo. El techo de aluminio brinda una gran resistencia a los daños que puede sufrir, al estar en el exterior. Muy difícilmente se puede encontrar grietas en poco tiempo o deformaciones. 


\section{Canaleta para lluvia}

El objetivo de la canaleta es llevar el agua de lluvia desde el techo hasta el bajante (cadena) para alcanzar un lugar específico en el suelo. Su función principal es proteger la estación de carga de problemas relacionados con la humedad en el techo, el cielo falso y los cimientos.

Figura 3. Instalación de la canaleta en el área perimetral.

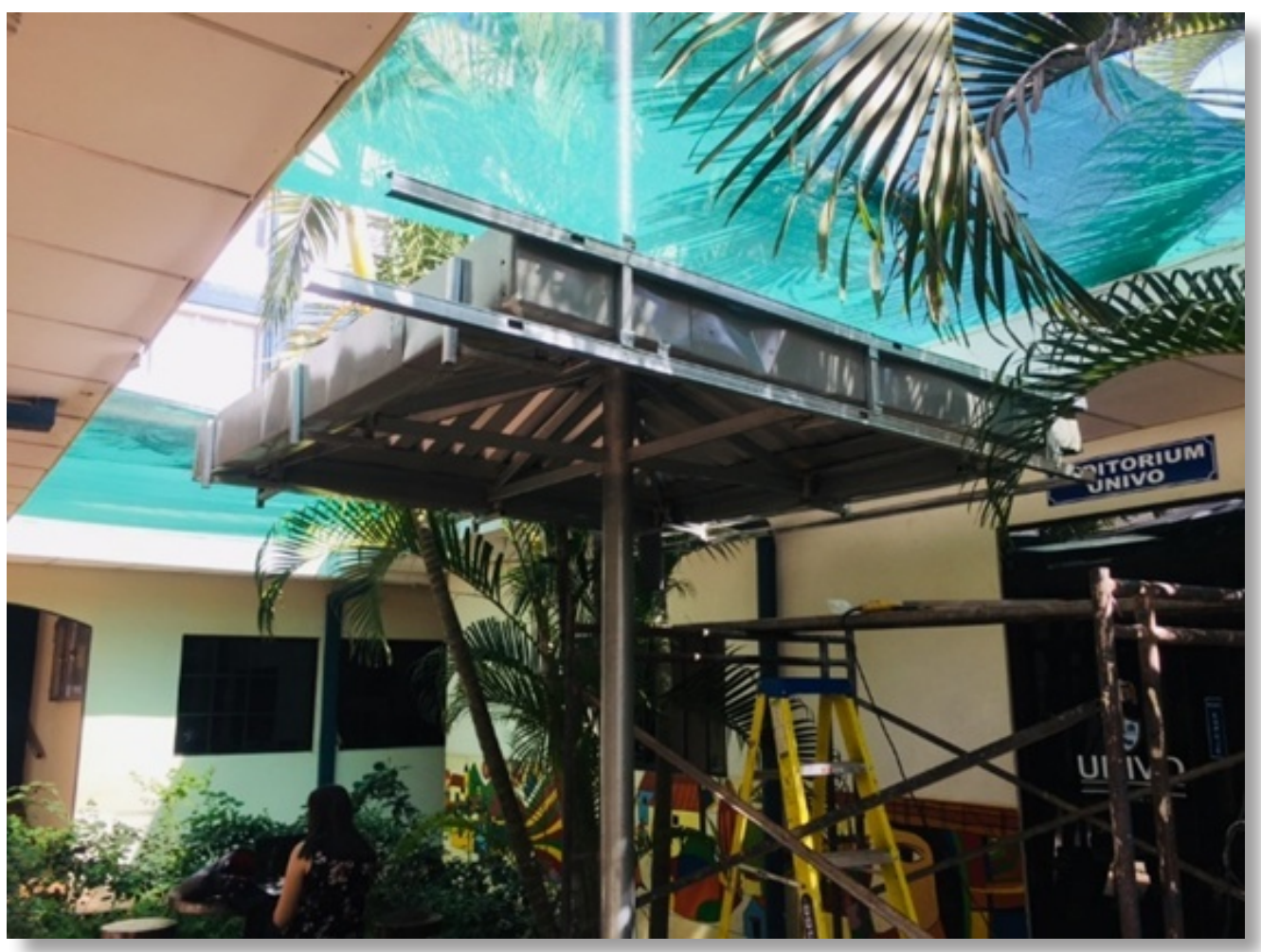

Fuente: Elaboración propia

\section{Fascia y cielo falso}

La fascia es una valla publicitaria para colocar en el diseño en vinil $2.40 \times 0.30 \mathrm{~m} 2 \mathrm{el}$ nombre del proyecto y para que tenga una mejor vistosidad la estación de carga. La instalación del cielo falso de tabla roca, aparte de ser un elemento decorativo, sirve también como aislante térmico y optimiza la iluminación interna (ojos de buey led) para los usuarios de la estación de carga.

\section{Mesa y sillas}

La estructura para el ensamblaje de la mesa de madera se hizo con tubo galvanizado de $|x|$ " para mayor resistencia en cuanto a peso. Para las sillas, se utilizó tubo de chapa I4. El ensamblaje de la mesa y las 6 sillas se realizó con madera de cedro recubierta con barniz marino (madera) para darle mayor duración y un excelente acabado decorativo. 
Figura 4. Finalización del quiosco, techo con cielo falso y fascia publicitaria. Mesa y sillas de madera encajada en la baldosa. La caja cuadrada en el centro de la mesa es donde se colocarán los 4 tomacorrientes dobles (uno por cada lado).



Fuente: Elaboración propia

\section{Cálculos y equipos del sistema y fotovoltaico}

Los cálculos y la ejecución del sistema FV fueron implementados por estudiantes de la carrera de Eficiencia Energética de la Universidad de Oriente, capacitados y evaluados por un asesor experto y con amplia experiencia en instalaciones fotovoltaicas.

\section{Cálculos para el dimensionamiento de la instalación fotovoltaica}

Para una instalación de energía solar fotovoltaica autónoma (aislada de la red eléctrica), es fundamental un correcto dimensionamiento tanto para poder abastecer con garantías la demanda energética que tengamos, como también para acotar el costo económico de la instalación, en este caso, la creación de una estación de carga para teléfonos móviles y laptops. Esto lo calcularemos en los siguientes pasos: 


\section{Paso I. Consumo de los equipos en corriente alterna}

Se calculará los consumos estimados de los dispositivos que se conectarán a la estación de carga.

\begin{tabular}{|l|c|c|c|c|c|}
\multicolumn{1}{|c|}{ Equipo } & $\begin{array}{c}\text { Potencia } \\
\text { en watts }\end{array}$ & $\begin{array}{c}\text { Equipos } \\
\text { simultáneos }(\mathbf{N})\end{array}$ & $\begin{array}{c}\text { Potencia total } \\
(\mathbf{W N})\end{array}$ & $\begin{array}{c}\text { Tiempo en } \\
\text { h/día (H) }\end{array}$ & $\begin{array}{c}\text { Consumo } \\
\text { Wh/día (WHN) }\end{array}$ \\
\hline $\begin{array}{l}\text { Tomacorriente } \\
\text { dobles con USB }\end{array}$ & 200 & 4 & 800 & 1.40 & II20 \\
\hline $\begin{array}{l}\text { Ojos de buey } \\
\text { TOTAL }\end{array}$ & 6 & 2 & 12 & 10 & I20 \\
\hline
\end{tabular}

Fuente: Vásquez (20I5).

El consumo de energía es de 1240 WHN (Wh/día).

\section{Paso 2. Eficiencia del inversor}

El inversor no solo tiene la función de conversión de CA-CC, sino que también la función de maximizar el rendimiento de la batería solar y la función de protección contra fallas del sistema. Para calcular la eficiencia del inversor, se realiza la siguiente fórmula:

$E T=E C A / I-10 \%$

En donde:

$\mathrm{ET}=$ energía total

$\mathrm{ECA}=$ energía de corriente alterna

ET $=1377.77778$ El $10 \%$ es la pérdida del inversor. Eso quiere decir que el inversor tiene una eficiencia del $90 \%$.

\section{Paso 3. Cálculo de las horas sol pico (HSP)}

La hora solar pico (HSP) es la energía que recibimos en horas por $\mathrm{m} 2$, y esta energía no es la misma dependiendo de la localización (cuanto más cerca del ecuador, mayor será) y por su época del año. Los rayos solares no son los mismos en un día de invierno que de verano en una localidad específica. Esta es la fórmula para calcular las horas sol pico:

HSP = radiación solar tablas / I Kw / m2

Cálculo para San Miguel:

$\mathrm{HSP}=5.29 \mathrm{KwH} / \mathrm{m} 2 / \mathrm{D}$

HSP $=5.29 \mathrm{H}$

Paso 4. Cálculo del rendimiento 


\begin{tabular}{|c|c|c|c|}
\hline Variable & Valor & Valor & Observaciones \\
\hline R & 0.75 & $75 \%$ & $75 \%$ \\
\hline KA & 0.005 & $0.50 \%$ & Autodescarga diaria \\
\hline KB & 0.1 & $10 \%$ & Pérdidas en los convertidores \\
\hline KC & 0.05 & $5 \%$ & Pérdidas en el inversor \\
\hline KV & 0.1 & $10 \%$ & Otras pérdidas \\
\hline PD & 0.8 & $80 \%$ & Profundidad máxima de descarga \\
\hline N & 1 & 1 & Días de autonomía de la batería \\
\hline
\end{tabular}

Fuente: Vásquez (20I5).

En donde:

$\mathrm{R}=$ rendimiento

$\mathrm{KA}=$ autodescarga del acumulador

$\mathrm{KB}=$ pérdida por el rendimiento del acumulador

$\mathrm{KC}=$ pérdida en el inversor

$\mathrm{KV}=$ pérdidas varias

$\mathrm{PD}=$ profundidad de descarga del acumulador

$\mathrm{N}=$ número de días

\section{Paso 5. Cálculo de energía real}

\begin{tabular}{|c|c|}
\hline Variable & Valor \\
\hline E & 1653.33 \\
\hline ET & 1240.00 \\
\hline R & 0.75 \\
\hline
\end{tabular}

Fuente: Vásquez (20I5).

En donde:

$E=$ energía real

$E T$ = energía total

$\mathrm{R}=$ rendimiento 
Paso 6. Cálculo de acumuladores

\begin{tabular}{|c|c|c|c|}
\hline Variable & Valor & Temperatura & Observaciones \\
\hline CU & 137.78 & Ah & \\
\hline VNS & 12 & & Voltaje nominal del sistema \\
\hline $\mathbf{N}$ & I & & Números de autonomía \\
\hline $\mathbf{E}$ & 1653.33 & & \\
\hline C & 162.0915033 & Ah & \\
\hline PD & 0.8 & & Capacidad de descarga del $80 \%$ \\
\hline $\mathbf{K t}$ & 1.06 & & \\
\hline $\mathbf{K t}$ & Temp. nominal & $22^{\circ}$ & \\
\hline Kt & Temp min. trab. & $32^{\circ}$ & \\
\hline AS & 1 & Acumuladores en serie & \\
\hline VNS & 12 & & \\
\hline VNA & 12 & & \\
\hline AP & 1.08 & 1 & Acumuladores en paralelo \\
\hline C & 162.09 & & \\
\hline CNA & 150 & Ah & \\
\hline AT & 1 & & \\
\hline
\end{tabular}

Fuente: Vásquez (20I5).

En donde:

$\mathrm{CU}=$ capacidad útil

VNS = voltaje nominal del sistema

$\mathrm{N}=$ número de días

$E=$ energía real

$\mathrm{C}=$ capacidad total

PD $=$ profundidad de descarga del acumulador

$\mathrm{KT}=$ factor de corrección de temperatura

AS $=$ acumuladores en serie

VNS = voltaje nominal del sistema

VNA = voltaje nominal del acumulador

$\mathrm{AP}=$ acumuladores en paralelo

$\mathrm{C}=$ capacidad total

$\mathrm{CNA}=$ capacidad nominal del acumulador

AT $=$ acumulador total 
Paso 7. Cálculo del generador fotovoltaico

\begin{tabular}{|c|c|c|c|}
\hline Variable & Valor & Observaciones & \\
\hline EG & 1837.04 & & \\
\hline E & 1653.33 & & \\
\hline NR & 0.9 & $\begin{array}{l}\text { Rendimiento } \\
\text { del regulador } \\
\text { en } \%\end{array}$ & \\
\hline PN & 385.85 & & \\
\hline HSP & 5.29 & & \\
\hline MS & 0.321543408 & \multicolumn{2}{|l|}{ Módulo en serie I } \\
\hline VNS & 12 & \multicolumn{2}{|c|}{ Tensión nominal del trabajo de la instalación } \\
\hline VNM & 37.32 & \multicolumn{2}{|c|}{ Tensión nominal del módulo elegido } \\
\hline MP & 1.10 & 1 & Panel en paralelo \\
\hline PN & 385.85 & & \\
\hline MS & 1 & & \\
\hline PP & 350 & \multicolumn{2}{|c|}{ Potencial nominal del panel elegido } \\
\hline MT & 1 & \multicolumn{2}{|l|}{ Módulos en total } \\
\hline PNI & 350 & & \\
\hline MT & 1 & & \\
\hline PP & 350 & & \\
\hline
\end{tabular}

Fuente: Vásquez (20I5).

En donde:

$E G=$ energía del generador fotovoltaico

$E=$ energía real

$N R=$ rendimiento del regulador

$\mathrm{PN}=$ potencia nominal del generador

$\mathrm{HSP}=$ hora sol pico

$\mathrm{MS}=$ módulos en serie

VNS = voltaje nominal del sistema

$\mathrm{MP}=$ módulos en paralelo

PP $=$ potencia pico del módulo

$M T=$ total de módulos

$\mathrm{PNI}=$ potencia nominal del inversor 


\section{Paso 8. Cálculo del regulador}

\begin{tabular}{|c|r|l|}
\hline Variable & Valor & Observaciones \\
\hline IPG & 9.2 & \\
\hline MP & I & \\
\hline IP & 9.2 & Sale de las especificaciones del panel IMP \\
\hline IR & 11.5 & Corriente del regulador \\
\hline IPG & 9.2 & \\
\hline
\end{tabular}

Fuente: Vásquez (20I5).

En donde:

IPG = corriente pico del generador

$M P=$ módulos en paralelo

$\mathrm{IP}=$ corriente pico

$\mathrm{IR}=$ corriente del regulador

IPG = corriente pico del generador

\section{Paso 9. Cálculo del inversor}

\begin{tabular}{|r|r|r|r|}
\hline Variable & Valor & \\
\hline PNI & 1377.78 & $1.38 \mathrm{Kw}$ & I inversor de I500w \\
\hline PS & 1240.00 & & \\
\hline N & 0.9 & & \\
\hline
\end{tabular}

Fuente: Vásquez (20I5).

En donde:

$\mathrm{PNI}=$ potencia nominal del inversor

$\mathrm{PS}=$ potencia de consumo mayor

$\mathrm{N}=$ número de días

\section{Análisis de cargas que se alimentarán con el sistema fotovoltaico}

- Tomacorriente dobles de 120 voltios AC polarizados con puerto USB

- Luminarias led (ojos de buey) de 6 watts; I 20 voltios AC cada uno

- Energía requerida a alimentar: I.24 Kwh/d

- Banco de acumuladores: una batería de I50 A-H: I 2 voltios DC

- Panel fotovoltaico: un panel solar fotovoltaico monocristalino de 350 watts pico; 37.32 voltios máximos

- Inversor: un inversor de onda sinusoidal pura. Entrada de 12 voltios y salida de 1500 watts; I 20 voltios 


\section{Diagrama de bloque}

Figura 5. En el diagrama representan de forma gráfica las relaciones existentes entre los diversos componentes del sistema fotovoltaico.

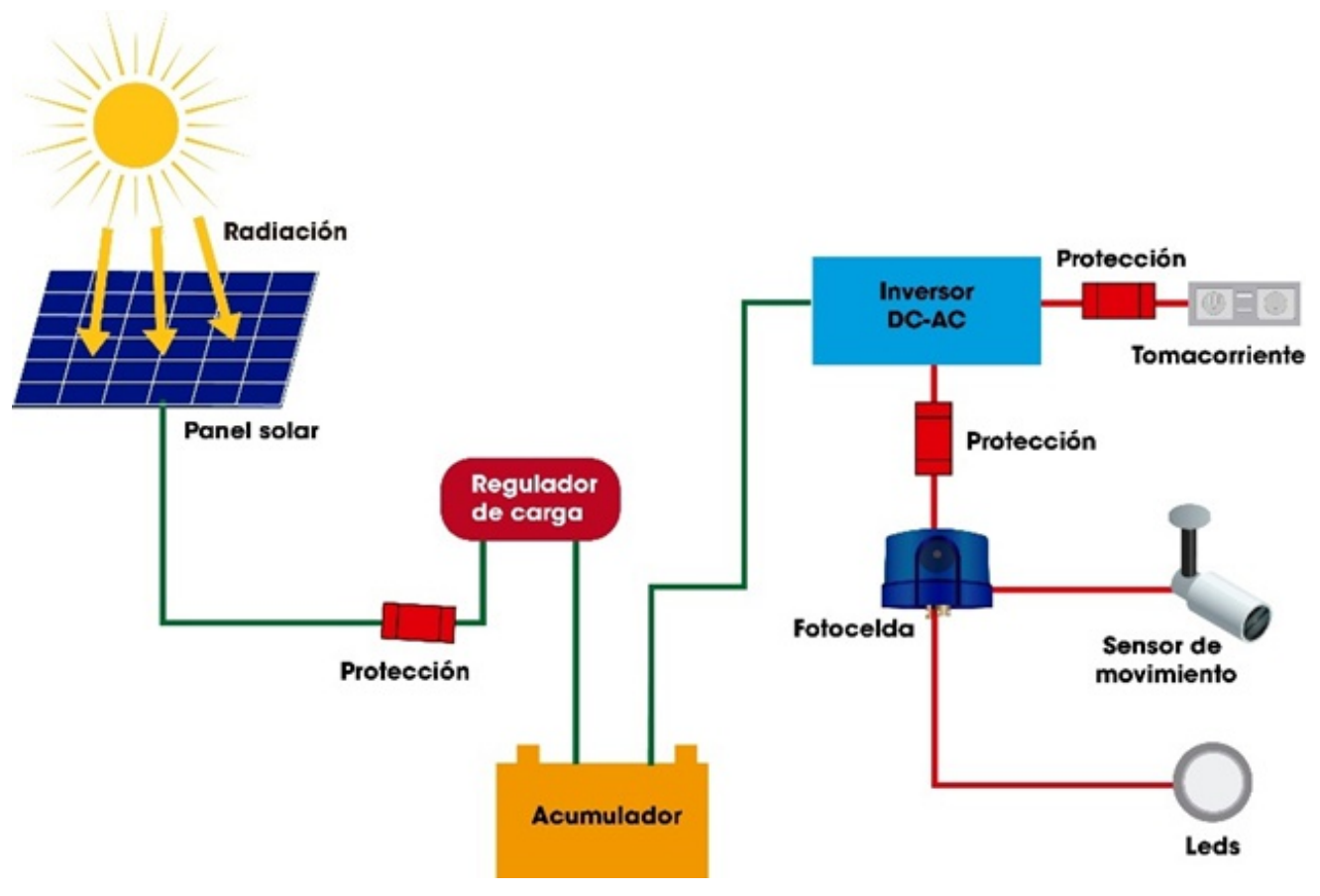

Fuente: Elaboración propia.

De acuerdo con el diagrama de bloque, el funcionamiento será de la siguiente forma:

I. La radiación solar incide sobre la placa fotovoltaica.

2. Las células del panel fotovoltaico convierten esa radiación en electricidad (en corriente continua $[\mathrm{CC}])$.

3. Un regulador de carga solar se coloca entre el campo fotovoltaico y el campo de baterías y básicamente se encarga de controlar el flujo de energía que circula entre ambos equipos.

4. La corriente continua va a un inversor solar, que la convierte en corriente alterna (AC), que es la que usamos para alimentar cualquier dispositivo electrónico, a 120 ○ 240 voltios.

5. Esa corriente alterna entra a la caja de tomacorrientes y led luminarios.

6. La electricidad, entonces, se distribuye en función de los consumos demandados por teléfonos celulares y laptops.

7. Las luminarias led se encenderán al pasar por dos filtros: primero, que la fotocelda no reciba luz solar (que esté de noche), y, segundo, que haya movimiento a 6 metros de la estación de carga. De lo contrario, permanecerán apagadas. 


\section{Descripción y selección de materiales utilizados}

"Panel solar monocristalino 350 W con dimensiones de 1956 × 992 x 40 mm. Este cuenta con unas excelentes características técnicas para climas que habitualmente tienen nubes, tormentas y temperaturas altas; esto es debido a que la sensibilidad del panel solar monocristalino consigue la máxima radiación solar en bajas temperaturas y resiste demasiado bien el sobrecalentamiento.

Batería seleccionada LTH (L-27MDC-160) ciclo profundo. Los acumuladores LTH ciclado profundo para aplicaciones fotovoltaicas han sido diseñados para soportar un alto número de descargas profundas y ser recargados sin afectar su desempeño.

Controlador de carga seleccionado (MPPT) 100/50 (punto de máxima potencia). Son más sofisticados electrónicamente, pues ajustan las tasas de carga según el nivel de la batería para permitir la carga más cerca de su capacidad máxima. También pueden controlar la temperatura de la batería para evitar el sobrecalentamiento.

Inversor N.E.K. DC - AC I2V/ I20V. I500W (onda pura). El inversor de corriente es un aparato electrónico cuya función es cambiar un voltaje de entrada de corriente continua (CC) a un voltaje simétrico de salida de corriente alterna (AC). Posee función de desconexión por sobrecarga o exceso de temperatura, por bajo o alto voltaje.

\section{Implementación del sistema eléctrico y fotovoltaico}

Para el encaje del panel solar, se hizo un marco rectangular con pernos para mayor protección y seguridad del panel solar fotovoltaico. Además, se colocó una abrazadera en el centro, ya que es imprescindible para la inclinación y la orientación en el ángulo azimut que se necesita a la hora de aprovechar al máximo el potencial de la instalación solar.

\section{Modificación de la caja marina}

La caja marina es donde irá la batería, el inversor, el control de carga y el cableado eléctrico; por lo tanto, necesita ventilación, ya que la alta temperatura incide en la variación del rendimiento de los equipos y su longevidad. Por ello se le hicieron dos aberturas con rejillas de ventilación, las cuales ayudarán a bajar la temperatura del interior de la caja.

\section{Polarización con barra cooper y cepo}

La polarización ayudará a los dispositivos y equipos a minimizar la posibilidad de recibir una descarga eléctrica cuando se tocan las partes metálicas de los dispositivos que han entrado en 
contacto con la electricidad.

\section{Instalación de fotocelda}

La ubicación de la fotocelda en la parte superior se utilizará como componente electrónico cuya resistencia disminuye con el aumento de intensidad de luz incidente. Es decir, que cuando no haya luz solar, encenderá las lámparas led.

\section{Conexión de inversor, batería y control de carga}

El inversor irá empernado en la parte superior al interior del gabinete o caja marina, donde la parte de los interruptores estará al lado de una de las rejillas para su ventilación. La ubicación de la batería irá en la parte inferior para descansar su peso. En la parte de la base de la caja, se debe quitar la tapa inferior y colocarle material de hule (de llanta) para aislar la temperatura y darle mejor ventilación. El control de carga irá empernado en la puerta de la caja para facilitar su conexión con el panel, el inversor y la batería.

La instalación eléctrica fotovoltaica se hará conectando elementos como la batería, el inversor, el regulador de voltaje, los cables y otros, que permitirán transformar la energía solar en una fuente de electricidad para alimentar los tomacorrientes.

Figura 6. Conexión de la fotocelda, inversor NEK, batería de ciclo profundo y control de carga MPPT. Vista aérea del panel solar. Abajo, la caja marina con las conexiones listas.
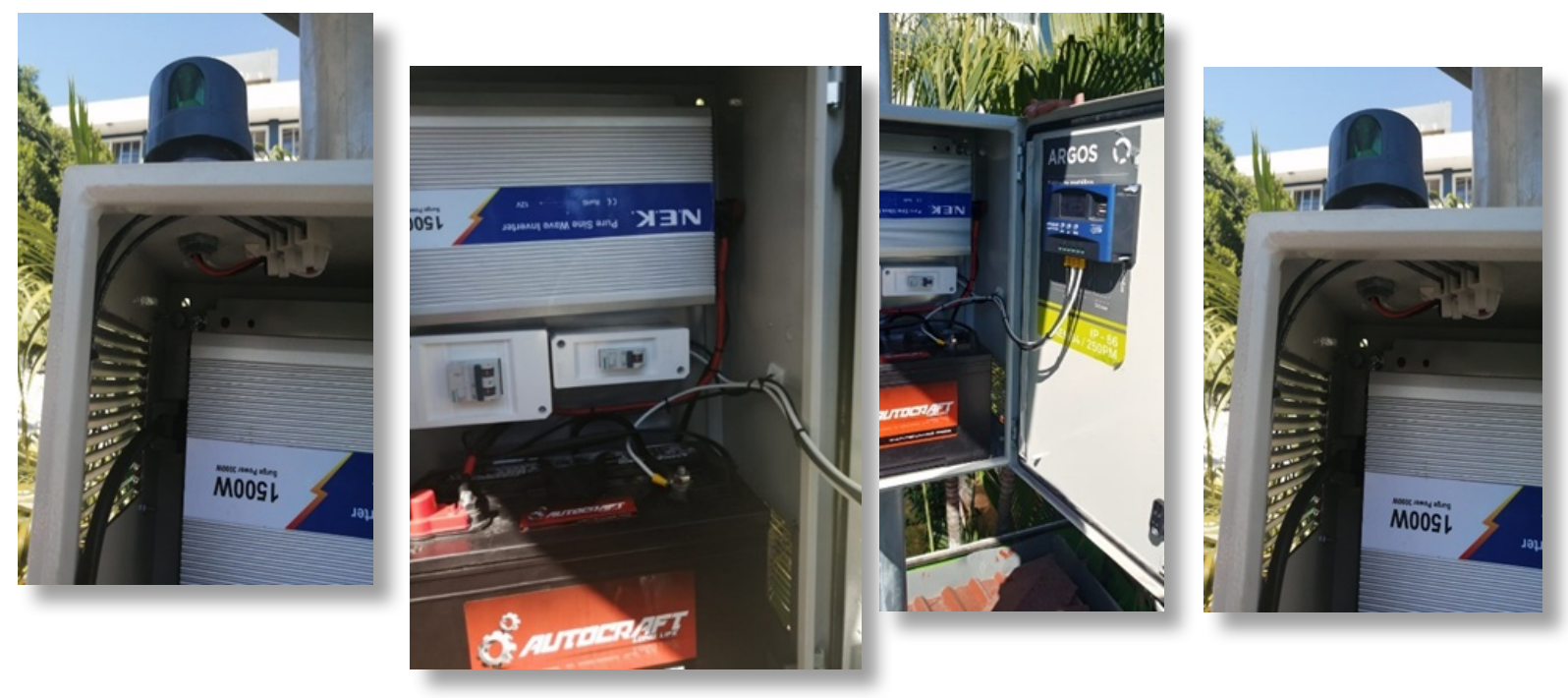

Fuente: Elaboración propia. 


\section{Instalación los tomacorrientes}

En la instalación de los 4 tomacorrientes dobles, primero debe de hacerse toda la conexión de los cables dentro de la caja de madera que está en el centro de la mesa. Los cables de los tomacorrientes se conectan directamente al positivo y al negativo de la instalación.

Dichos tomacorrientes se caracterizan por tener tres puntos de conexión; el de fase, vivo o positivo; el neutro o negativo, y el de tierra física. Es muy importante el uso de estos tomacorrientes dobles con 2 terminales USB cada uno.

\section{Adaptación de sensor de movimiento}

Se adaptó un sensor de movimiento a las luces led, con el objetivo de que puedan encenderse siempre y cuando haya movimiento a $6 \mathrm{~m}$ de distancia de detección, teniendo en cuenta también que la fotocelda no reciba luz solar.

El sensor de movimiento busca apuntar hacia la entrada del pasillo, teniendo un ángulo de 1800 y una distancia de detección de $6 \mathrm{~m}$. Las luces encenderán con 2 parámetros: la fotocelda a la que no le entre luz solar y el sensor de movimiento cuando los usuarios estén dentro o pasen en frente de la estación de carga. Esto les dará autonomía a las luces led.

\section{Resultados}

\section{Cálculo de consumo de corriente de los equipos en corriente alterna}

El cálculo se hace con el objetivo de hacer un estimado de consumo de corriente alterna de los aparatos eléctricos o electrónicos que se conectarán al sistema fotovoltaico.

\begin{tabular}{|l|c|c|c|c|c|c|c|}
\multicolumn{1}{|c|}{ Equipo } & $\begin{array}{c}\text { Potencia } \\
\text { en W }\end{array}$ & $\begin{array}{c}\text { Equipos } \\
\text { simultáneos }\end{array}$ & $\begin{array}{c}\text { Potencia } \\
\text { total }\end{array}$ & $\begin{array}{c}\text { Tiempo } \\
\text { h/día }\end{array}$ & $\begin{array}{c}\text { Consumo } \\
\text { wh/día }\end{array}$ & $\begin{array}{c}\text { Voltaje } \\
\text { (V) }\end{array}$ & $\begin{array}{c}\text { Corriente } \\
\text { total (A) }\end{array}$ \\
\hline $\begin{array}{l}\text { Tomacorrientes } \\
\text { dobles }\end{array}$ & 200 & 4 & 800 & 1.40 & 1120 & 120 & 6.67 \\
\hline Ojos de buey & 6 & 2 & 12 & 10.0 & 120 & 120 & 0.1 \\
\hline TOTAL & & & $\mathbf{8 I 2}$ & & 1240 & & $\mathbf{6 . 7 7}$ \\
\hline
\end{tabular}




\section{Resultados de probar voltaje (V) y corriente (A) del sistema FV}

Se realizaron pruebas en un período de 16 días no continuos, probando el voltaje de la batería y el voltaje del inversor, para calcular la corriente que genera la estación de carga.

\begin{tabular}{|c|c|c|c|c|}
\hline Día & $\begin{array}{l}\text { Voltaje } \\
\text { batería } \\
\text { (V) }\end{array}$ & $\begin{array}{l}\text { Voltaje } \\
\text { del } \\
\text { Inversor } \\
\text { (V) }\end{array}$ & $\begin{array}{c}\text { Potencia } \\
\text { total } \\
\text { (W) }\end{array}$ & Corriente (A) \\
\hline $06 / 01 / 2020$ & 13.6 & 121 & 812 & 6.71 \\
\hline $14 / 01 / 2020$ & 12 & 120 & 812 & 6.77 \\
\hline $17 / 01 / 2020$ & 12 & 120 & 812 & 6.77 \\
\hline $21 / 01 / 2020$ & 12 & 120 & 812 & 6.77 \\
\hline $25 / 01 / 2020$ & 13.6 & 121 & 812 & 6.71 \\
\hline $30 / 01 / 2020$ & 12 & 120 & 812 & 6.77 \\
\hline $04 / 02 / 2020$ & 12 & 120 & 812 & 6.77 \\
\hline $08 / 02 / 2020$ & 12 & 120 & 812 & 6.77 \\
\hline $11 / 02 / 2020$ & 13 & 121 & 812 & 6.71 \\
\hline $20 / 02 / 2020$ & 13 & 121 & 812 & 6.71 \\
\hline $26 / 02 / 2020$ & 12 & 120 & 812 & 6.77 \\
\hline $03 / 03 / 2020$ & 12 & 120 & 812 & 6.77 \\
\hline $10 / 03 / 2020$ & 12 & 120 & 812 & 6.77 \\
\hline $14 / 03 / 2020$ & 13 & $|2|$ & 812 & 6.77 \\
\hline $17 / 03 / 2020$ & 12 & 120 & 812 & 6.77 \\
\hline $21 / 03 / 2020$ & 13 & 121 & 812 & 6.71 \\
\hline
\end{tabular}

Fuente: Elaboración propia

Comportamiento del voltaje y la corriente de la batería e inversor

Gráfico I. Comportamiento del voltaje en 16 días discontinuos.



Fuente: Elaboración propia 
Gráfico 2. Comportamiento de la corriente total en 16 días discontinuos.

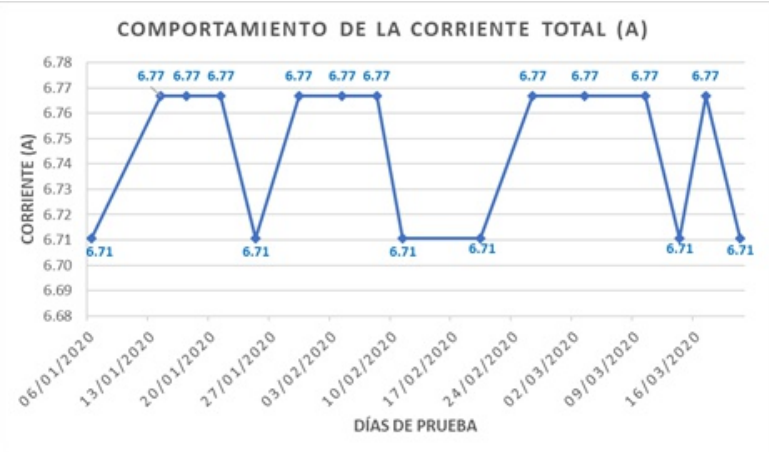

Fuente: Elaboración propia

\section{Resultados de conectar de I a 8 laptops al sistema fotovoltaico}

Se realizó una conexión múltiple con 8 laptops para evaluar su voltaje y la corriente total de acuerdo con los cálculos establecidos previamente, haciendo referencia a los 4 tomacorrientes dobles para enchufar los cargadores de laptops. Los resultados fueron los siguientes:

Tabla 10: Capacidad en potencia y corriente de I a 8 laptops conectadas

\begin{tabular}{|c|c|c|c|c|c|c|}
\hline $\begin{array}{c}\text { Cantidad de laptops } \\
\text { conectadas }\end{array}$ & $\begin{array}{c}\text { Voltaje } \\
\text { batería }\end{array}$ & $\begin{array}{c}\text { Voltaje } \\
\text { inversor }\end{array}$ & $\begin{array}{c}\text { Potencia } \\
\text { unitaria (W) }\end{array}$ & $\begin{array}{c}\text { Potencia } \\
\text { total } \\
\text { (W) }\end{array}$ & $\begin{array}{c}\text { Corriente } \\
\text { unitaria (A) }\end{array}$ & $\begin{array}{c}\text { Corriente } \\
\text { total (A) }\end{array}$ \\
\hline I laptop & 12 & 120 & 65 & 65 & 0.54 & $\mathbf{0 . 5 4}$ \\
\hline 2 laptops & 12 & 120 & 65 & 130 & 0.54 & $\mathbf{1 . 0 8}$ \\
\hline 3 laptops & 12 & 120 & 65 & 195 & 0.54 & $\mathbf{1 . 6 3}$ \\
\hline 4 laptops & 12 & 120 & 65 & $\mathbf{2 6 0}$ & 0.54 & $\mathbf{2 . 1 7}$ \\
\hline 5 laptops & 12 & 120 & 65 & 325 & 0.54 & $\mathbf{2 . 7 1}$ \\
\hline 6 laptops & 12 & 120 & 65 & 390 & 0.54 & $\mathbf{3 . 2 5}$ \\
\hline 7 laptops & 12 & 120 & 65 & $\mathbf{4 5 5}$ & 0.54 & $\mathbf{3 . 7 9}$ \\
\hline 8 laptops & 12 & 120 & 65 & $\mathbf{5 2 0}$ & 0.54 & $\mathbf{4 . 3 3}$ \\
\hline
\end{tabular}

Fuente: Elaboración propia

Gráfico 3. Comportamiento de la potencia total conectando de I a 8 laptops.

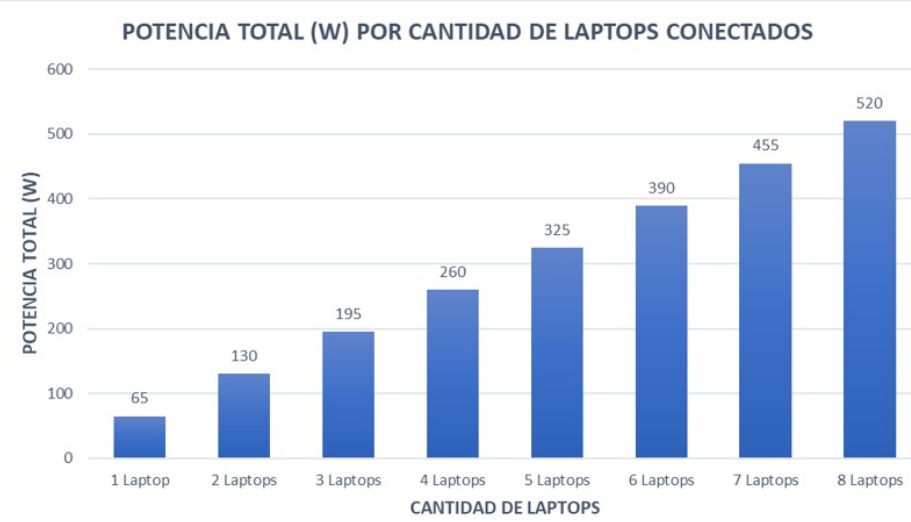

Fuente: Elaboración propia 
Gráfico 4. Comportamiento de la corriente total conectando de I a 8 laptops.

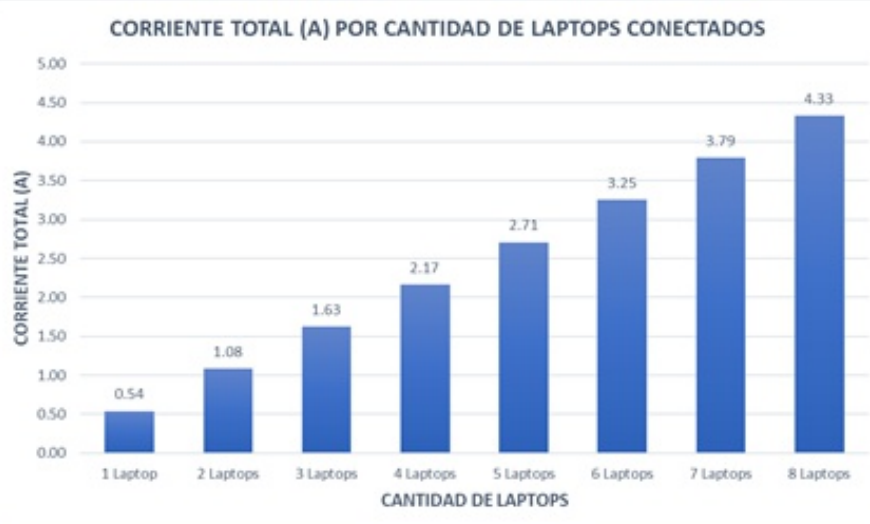

Fuente: Elaboración propia

\section{Resultado de conectar de I a I 6 teléfonos móviles al sistema fotovoltaico}

Se conectaron 16 teléfonos móviles. La estación de carga posee 4 placas: cada una posee 2 terminales para conector de cubo y 2 puertos USB para conectar solo el cable. Esto significa que todos los tomacorrientes están ocupados.

\begin{tabular}{|c|c|c|c|c|c|c|}
\hline $\begin{array}{c}\text { Cantidad de } \\
\text { dispositivos } \\
\text { móviles }\end{array}$ & $\begin{array}{c}\text { Voltaje } \\
\text { batería }\end{array}$ & Voltaje inversor & $\begin{array}{c}\text { Potencia } \\
\text { unitaria } \\
\mathbf{( W )}\end{array}$ & $\begin{array}{c}\text { Potencia } \\
\text { total }(\mathbf{W})\end{array}$ & $\begin{array}{c}\text { Corriente } \\
\text { unitaria (A) }\end{array}$ & $\begin{array}{c}\text { Corriente } \\
\text { total (A) }\end{array}$ \\
\hline $\mathbf{I}$ & 12 & 120 & 15 & 15 & 0.13 & 0.13 \\
\hline $\mathbf{2}$ & 12 & 120 & 15 & 30 & 0.13 & 0.25 \\
\hline $\mathbf{3}$ & 12 & 120 & 15 & 45 & 0.13 & 0.38 \\
\hline $\mathbf{4}$ & 12 & 120 & 15 & 60 & 0.13 & 0.50 \\
\hline $\mathbf{5}$ & 12 & 120 & 15 & 75 & 0.13 & 0.63 \\
\hline $\mathbf{6}$ & 12 & 120 & 15 & 90 & 0.13 & 0.75 \\
\hline $\mathbf{7}$ & 12 & 120 & 15 & 105 & 0.13 & 0.88 \\
\hline $\mathbf{8}$ & 12 & 120 & 15 & 120 & 0.13 & 1.00 \\
\hline $\mathbf{9}$ & 12 & 120 & 15 & 135 & 0.13 & 1.13 \\
\hline $\mathbf{1 0}$ & 12 & 120 & 15 & 150 & 0.13 & 1.25 \\
\hline $\mathbf{I 1}$ & 12 & 120 & 15 & 165 & 0.13 & 1.38 \\
\hline $\mathbf{1 2}$ & 12 & 120 & 15 & 180 & 0.13 & 1.50 \\
\hline 13 & 12 & 120 & 15 & 195 & 0.13 & 1.63 \\
\hline 14 & 12 & 120 & 15 & 210 & 0.13 & 1.75 \\
\hline 15 & 12 & 120 & 15 & 225 & 0.13 & 1.88 \\
\hline 16 & 12 & 120 & 15 & 240 & 0.13 & 2.00 \\
\hline
\end{tabular}

Fuente: Elaboración propia 


\section{Capacidad de la potencia y corriente de I a I 6 teléfonos conectados}

Gráfico 5. Comportamiento de la potencia total conectando de I a 16 teléfonos móviles.



Fuente: Elaboración propia

Gráfico 6. Comportamiento de la corriente total conectando de I a I6 teléfonos móviles.

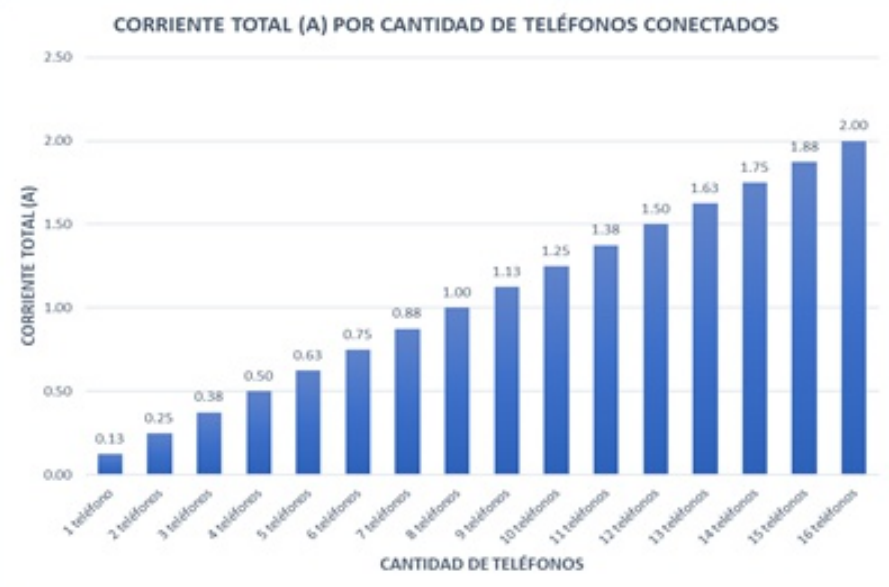

Fuente: Elaboración propia

Se hizo otra prueba con 6 estudiantes, los cuales ocuparon las 6 sillas con las que cuenta la estación de carga. Cada estudiante poseía una laptop y un teléfono celular para hacer uso de la energía renovable. Los resultados de su corriente total fueron los siguientes:

\begin{tabular}{|c|c|c|c|c|c|c|}
\hline $\begin{array}{c}\text { Cantidad de } \\
\text { laptops y celulares }\end{array}$ & $\begin{array}{l}\text { Voltaje } \\
\text { batería }\end{array}$ & Voltaje inversor & $\begin{array}{l}\text { Potencia } \\
\text { unitaria } \\
\text { (W) }\end{array}$ & $\begin{array}{l}\text { Potencia } \\
\text { total } \\
\text { (W) }\end{array}$ & $\begin{array}{l}\text { Corrient } \\
\text { e unitaria } \\
\text { (A) }\end{array}$ & $\begin{array}{l}\text { Corrient } \\
\text { e total } \\
\text { (A) }\end{array}$ \\
\hline \multicolumn{7}{|l|}{ Laptops } \\
\hline 6 & 12 & 120 & 65 & 390 & 0.54 & 3.25 \\
\hline \multicolumn{7}{|l|}{ Teléfonos } \\
\hline 6 & 12 & 120 & 15 & 90 & 0.13 & 0.75 \\
\hline & TOTAL & & & 480 & & 4.00 \\
\hline
\end{tabular}

Elaboración propia 


\section{Capacidad de la potencia y corriente con 6 laptops y 6 teléfonos conectados}

Gráfico 7. Comportamiento de la potencia total conectando 6 laptops y 6 teléfonos móviles.



Fuente: Elaboración propia

Gráfico 8. Comportamiento de la corriente total conectando 6 laptops y 6 teléfonos móviles.



Fuente: Elaboración propia

\section{Discusión}

Para la construcción de un sistema solar fotovoltaico, fue necesario hacer el cálculo de la corriente alterna total. Para ello se hizo una tabla, calculando los consumos estimados de corriente alterna de los aparatos eléctricos o electrónicos que se conectarán al sistema fotovoltaico.

En la tabla 8, se colocaron las potencias de los 4 tomacorrientes con potencia de $200 \mathrm{~W}$, y las 2 lámparas led (ojos de buey) de $6 \mathrm{~W}$ cada una. Para el cálculo, se multiplica la potencia por la cantidad de dispositivos y así obtener la potencia total. El dato que nos interesa es la suma 
de las potencias totales de los 4 tomacorrientes con las 2 luminarias led:

PTTOMACORRIENTES $=4 \times 200 \mathrm{~W}=800 \mathrm{~W}$

PTLED $=2 \times 6=12 \mathrm{~W}$

$\mathrm{PT}=800 \mathrm{~W}+12 \mathrm{~W}$

$\mathrm{PT}=812 \mathrm{~W}$

Luego de tener la potencia total (PT) de los dos dispositivos, se procede a calcular la corriente total, teniendo en cuenta que el voltaje total es de 120 y la fórmula para calcular la corriente es $\mathrm{I}=\mathrm{P} / \mathrm{V}$. El resultado será en amperios $(\mathrm{A})$.

ITOTAL $=812 \mathrm{~W} / 120 \mathrm{~V}$

ITOTAL $=6.77 \mathrm{~A}$

Esto significa que el sistema fotovoltaico está capacitado para suministrar corriente de 6.77 A. En el caso de conectarle uno o varios dispositivos eléctricos o electrónicos que tengan una corriente superior al que está diseñado, el sistema fotovoltaico se autoprotegerá encendiendo una alarma y cortando el paso de corriente para evitar cortocircuitos y causar daño al equipo fotovoltaico.

\section{Análisis de probar voltaje (V) y corriente (A) del sistema FV}

Se realizaron pruebas en un período de 16 días no continuos para probar el voltaje de la batería y el voltaje del inversor, a fin de calcular la corriente que genera la estación de carga.

De acuerdo con la gráfica I, el voltaje que genera la batería a $12 \mathrm{~V}$ a $13.6 \mathrm{~V}$ es de corriente directa (CD). Luego, el inversor la convierte a corriente alterna $(C A)$ de $120 \mathrm{~V}$ a $12 \mathrm{IV}$, suministra a las fuentes de distribución (tomacorrientes) y alimenta las cargas.

En la gráfica 2, la corriente total se mantiene entre 6.77 A y 6.7I A. Cada dispositivo que le conectan, entre laptops y teléfono móvil, hacen variar su potencia $(A)$ en cada uno de ellos. Para saber la potencia de estos dispositivos, en las etiquetas de los cargadores dice el $\mathrm{V}=$ voltaje, la $P=$ potencia en $W$ (watts o vatios) y sus $A=$ amperios. Por ejemplo, algunas laptops HP traen $V=18.5, P=65 \mathrm{~W}$, y si se necesita saber su corriente, se hace la operación $\mathrm{I}=\mathrm{P} / \mathrm{V}$, que sería:

ILAPTOP $=65$ WLAPTOP $/$ I8.5 VLAPTOP

ILAPTOP $=3.5 \mathrm{~A}$

Cabe hacer la mención de que no todas las laptops cuentan con estas características; tiene mucho que ver su modelo y su año de ensamblaje. 


\section{Potencia total de las 8 laptops}

De acuerdo con los resultados obtenidos en la gráfica 3, al tener conectados todos tomacorrientes con los 8 equipos, con $65 \mathrm{~W}$ por cada laptop, se alcanza una potencia total de la cantidad de laptops:

PTTOTAL LAPTOPS $=65$ WPOTENCIA UNITARIA LAPTOP $\times 8$ LAPTOS

PTTOTAL 8 LAPTOPS $=520 \mathrm{~W}$

Los $520 \mathrm{~W}$ se encuentran dentro de la capacidad de la estación de carga, ya que la máxima potencia que posee es de $812 \mathrm{~W}$, de acuerdo con los cálculos establecidos previamente.

\section{Corriente total de las 8 laptops}

La corriente por cada laptop es de $0.54 \mathrm{~A}$. Al conectar las 8 laptops, la corriente total (IT) sería:

ITTOTAL 8 LAPTOPS $=0.54$ WPOTENCIA UNITARIA LAPTOP $\times$ 8LAPTOS

ITTOTAL 8 LAPTOPS $=4.33 \mathrm{~A}$

El total de la corriente es de $4.33 \mathrm{~A}$, la cual se encuentra dentro de la capacidad de la estación de carga, ya que tiene una capacidad de $6.77 \mathrm{~A}$, de acuerdo con los cálculos establecidos previamente.

\section{Potencia total de los 16 teléfonos}

De acuerdo con el gráfico 5 , al tener conectados los 4 tomacorrientes dobles, incluyendo los puertos USB, se enchufaron 16 teléfonos móviles, con $15 \mathrm{~W}$ por cada smartphone. La potencia total (PT) sería:

PTTOTAL 16 TELÉFONOS = 15 WPOTENCIA UNITARIA TELÉFONO × 16 TELÉFONOS PTTOTAL 16 TELÉFONOS $=240 \mathrm{~W}$

Los $240 \mathrm{~W}$ de la potencia total de los 16 teléfonos se encuentran dentro de la capacidad de la estación de carga, ya que su máxima potencia es de $812 \mathrm{~W}$, de acuerdo con los cálculos establecidos previamente.

Corriente total de los 16 teléfonos

La corriente por cada teléfono es de 0.13 A. Para saber su corriente total (IT), se multiplica por los 16 teléfonos celulares conectados:

ITTOTAL 16 TELÉFONOS $=0.13$ WCORRIENTE UNITARIA TELÉFONO $\mathrm{x}$ I6TELÉFONOS

ITTOTAL 16 TELÉFONOS $=2.0 \mathrm{~A}$

De acuerdo con la gráfica 6 , la corriente que genera cada teléfono celular es de $0.13 \mathrm{~A}$; al 
conectar los 16 teléfonos, tenemos una corriente total (IT) 2.0A, la cual se encuentra dentro de la capacidad de la estación de carga, ya que tiene una capacidad de 6.77 A con los cálculos establecidos previamente.

\section{Potencia total de las 6 laptops}

De acuerdo con el gráfico 7, al tener conectadas las 6 laptops con $65 \mathrm{~W}$, se genera una potencia total de $390 \mathrm{~W}$. Hay que recordar que la potencia total es igual a la potencia unitaria por la cantidad de laptops.

PTTOTAL 6 LAPTOPS $=65$ WPOTENCIA UNITARIA LAPTOP $\times 6$ LAPTOPS

PTTOTAL 6 LAPTOPS $=390 \mathrm{~W}$

\section{Potencia total de los 6 teléfonos}

Por otra parte, los 6 teléfonos celulares, con $15 \mathrm{~W}$ por cada uno, alcanzan una potencia total de $90 \mathrm{~W}$.

PTTOTAL 6 TELÉFONOS $=15$ WPOTENCIA UNITARIA TELÉFONO $\times 6$ TELÉFONOS

PTTOTAL 6 TELÉFONOS $=90 \mathrm{~W}$

\section{Potencia total de las 6 laptops y los 6 teléfonos}

La suma de las potencias totales entre las 6 laptops y los 6 teléfonos es la siguiente:

PTTOTAL 6 LAPTOPS $=390 \mathrm{~W}$

PTTOTAL 6 TELÉFONOS $=90 \mathrm{~W}$

PTTOTAL 6 LAPTOPS Y 6 TELÉFONOS $=480 \mathrm{~W}$

Los 480W se encuentran dentro de la capacidad de la estación de carga, ya que su máxima potencia es $812 \mathrm{~W}$, de acuerdo con los cálculos expuestos anteriormente.

Corriente total de las 6 laptops

La corriente por cada laptop es de $0.54 \mathrm{~A}$. Al conectar las 6 laptops, la corriente total (IT) es la siguiente:

ITTOTAL 6 LAPTOPS $=0.54$ WPOTENCIA UNITARIA LAPTOP $\times$ 6LAPTOPS

ITTOTAL 6 LAPTOPS $=3.25 \mathrm{~A}$

\section{Corriente total de los 6 teléfonos}

La corriente por cada teléfono es de 0.13A. Para saber su corriente total (IT) con los 6 teléfonos celulares conectados, tenemos:

ITTOTAL 6 TELÉFONOS $=0.13$ WCORRIENTE UNITARIA TELÉFONO $\times$ 6TELÉFONOS

ITTOTAL 6 TELÉFONOS $=0.75 \mathrm{~A}$ 


\section{Corriente total de las 6 laptops y los 6 teléfonos}

La suma de las corrientes totales entre las 6 laptops y los 6 teléfonos es esta:

ITTOTAL 6 LAPTOPS $=3.25 \mathrm{~A}$

ITTOTAL 6 TELÉFONOS $=0.75 \mathrm{~A}$

ITTOTAL 6 LAPTOPS Y 6 TELÉFONOS $=4.0 \mathrm{~A}$

De acuerdo con la gráfica 8, al tener conectadas las 6 laptops, con $0.54 \mathrm{~A}$ cada una, hacen una corriente total de $3.25 \mathrm{~A}$. Por otra parte, los 6 teléfonos celulares, con $0.13 \mathrm{~A}$ por cada teléfono móvil, alcanzan una corriente total de $0.75 \mathrm{~A}$. La suma de las corrientes totales entre las 6 laptops y los 6 teléfonos es de 4.0 A. Ello se encuentra dentro de la capacidad de la estación de carga, ya que la corriente máxima es de $6.77 \mathrm{~A}$, de acuerdo con los cálculos establecidos anteriormente.

\section{Autoprotección de cortocircuitos}

La estación de carga está diseñada para satisfacer las conexiones múltiples de dispositivos móviles y laptops. De acuerdo con los cálculos que se hicieron al inicio del proyecto, en el caso de conectarle un dispositivo eléctrico o electrónico que tenga un voltaje superior al que está diseñado, el sistema fotovoltaico se autoprotegerá encendiendo una alarma y cortando el paso de corriente para evitar cortocircuitos y causar daño al equipo fotovoltaico.

Ahorro de energía eléctrica

El sistema fotovoltaico ha tenido una demanda bastante alta por parte de sus usuarios, técnicamente es viable y capaz de satisfacer al $100 \%$ como fuente de energía renovable, aportando, a su vez, un ahorro de energía de la red eléctrica de la Universidad de Oriente. A continuación, se calculará el ahorro aproximado y en cuánto tiempo se recuperará la inversión.

Consumo Whn $=1240$ Whn (de acuerdo con la tabla de cálculos de consumo)

Convertir a KWh (kilowatts hora)

KWhCONSUMO $=1240$ Whn $/ 1000$

$\mathrm{KWhCONSUMO}=1.24 \mathrm{KWh}$

Costo por KWh (EEO) $=\$ 0.1463$

KWhCOSTO CONSUMO HORA $=1.24 \mathrm{KWh} \times \$ 0.1463$

KWhCOSTO CONSUMO HORA $=\$ 0.18$ 


\begin{tabular}{|r|r|r|r|r|}
\hline Ahorro por hora & $\begin{array}{c}\text { Ahorro por } \\
\text { día }\end{array}$ & $\begin{array}{r}\text { Ahorro por } \\
\text { mes (30 días) }\end{array}$ & $\begin{array}{r}\text { Ahorro por año } \\
\text { (360 días) }\end{array}$ & Ahorro por 25 años \\
\hline$\$ 0.18$ & $\$ 4.35$ & $\$ 130.63$ & $\$ 1567.40$ & $\$ 39,184.99$ \\
\hline
\end{tabular}

Fuente: Elaboración propia

Calculando el período de recuperación de la inversión (PRI)

Inversión $=\$ 2116.39$

Ahorro al año $=\$ 1567.40$

$P R I=\$ 2 I I 6.39 / \$ 1567.40$

$\mathrm{PRI}=\mathrm{I} .35$

Lo anterior significa que el período de recuperación será de un año con cuatro meses, aproximadamente.

\section{Calculando el ahorro neto en 25 años}

Inversión $=\$ 2116.39$

Ahorro por 25 años $=\$ 37,497.60$ (tiempo estimado de vida de vida útil de un panel solar) AHORRO-NETO25 AÑOS $=\$ 37,497.60-\$ 2116.39$

AHORRO-NETO25 AÑOS $=\$ 35,381.21$

Figura 8. Entrega del prototipo de la estación de carga. Funcionamiento de día y de noche.

Fuente: fotografías propias, tomadas a estudiantes haciendo uso del cargador solar.
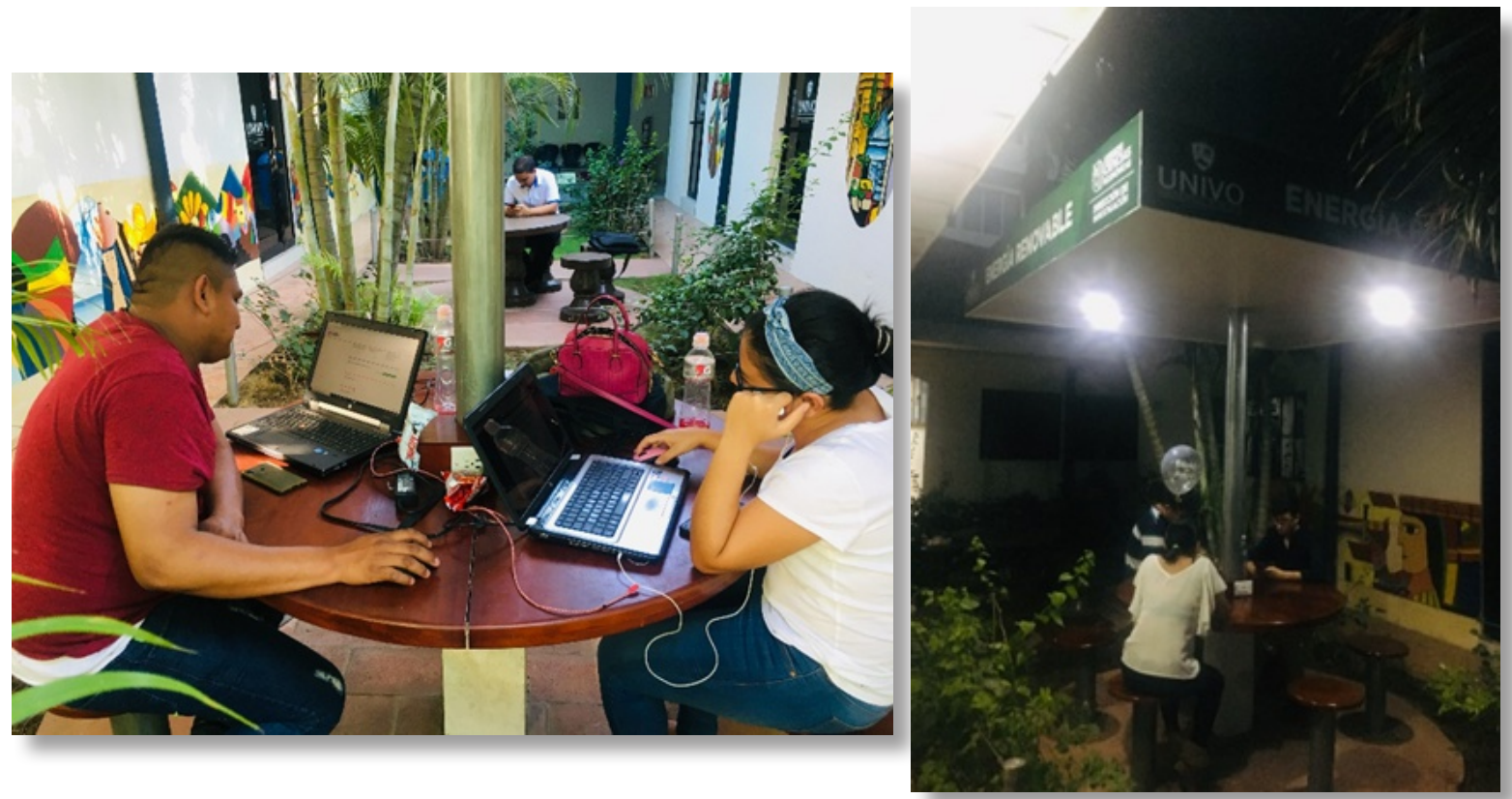

Fuente: Elaboración propia 


\section{Conclusiones}

El módulo fotovoltaico en la Universidad de Oriente es una fuente de energía ecológica renovable y tiene como recurso natural inagotable el sol. La ciudad de San Miguel, por estar una zona bastante cálida, tiene la bondad de tener bastante irradiancia solar que se está aprovechando sin afectar al medio ambiente. La estación de carga está diseñada para satisfacer las conexiones de múltiples dispositivos móviles y laptops, de acuerdo con los cálculos que se hicieron al inicio del proyecto.

Con la estación de carga fotovoltaica en la Universidad de Oriente (cede central), se le brinda un beneficio a los estudiantes, docentes, visitantes y personal de la institución, para que puedan recargar sus teléfonos móviles y laptops, quienes utilizan sus dispositivos para desarrollar clases virtuales (docentes y alumnos), investigaciones, tareas, entre otras actividades. De esa manera, se fomenta el uso de las energías renovables. Esta ha tenido una demanda bastante alta por parte de sus usuarios, debido a su llamativo diseño de mesa y sillas de madera en su estructura; además de ser capaz de satisfacer al $100 \%$ como fuente de energía renovable, por ser fuente limpia e inagotable, indispensable para combatir el cambio climático, aportando, a su vez, un ahorro de energía de la red eléctrica de la Universidad de Oriente.

\section{Bibliografía}

Casa, M. y Barrio, M. (2017). Instalaciones solares fotovoltaicas. Alfaomega Marcombo.

Creus, A. (2014). Energías renovables (2.a ed.). Ediciones de la U.

Juana Sardón, J. M. de (Coord.). (2003). Energías renovables para el desarrollo. Paraninfo.

Molina Roa, N. (2018). Taller de redacción de artículos para estudiantes universitarios. Universidad de La Salle.

Vásquez, M. C. (2015). Instalaciones solares fotovoltaicas (2.a ed.). Ediciones de la U.

Vega de Kuyper, J. C. y Ramírez Morales, S. (2015). Fuentes de energía, renovables y no renovables. Aplicaciones. Alfaomega Marcombo. 